International Journal of Distributed and Parallel Systems (IJDPS) Vol.3, No.5, September 2012

\title{
Cross-Layer Energy Optimization in Cooperative Cellular Systems
}

\author{
M.Sushanth Babu ${ }^{1}$ and K.kishan Rao ${ }^{2}$ \\ ${ }^{1}$ Department of Electronics \& Communication Engineering, \\ Vaagdevi College of Engineering, Jawaharlal Nehru Technological University, A.P., \\ INDIA. \\ ${ }^{1}$ sushanth_6@yahoo.com \\ ${ }^{2}$ Director, Department of Electronics \& Communication Engineering, \\ Vaagdevi College of Engineering, Jawaharlal Nehru Technological University, A.P., \\ INDIA. \\ ${ }^{2}$ prof_kkrerediffmail.com
}

\begin{abstract}
Cooperative networking is currently under standardization for future wireless cellular systems. This paper considers energy efficient transmission schemes in cooperative cellular transceiver systems. In which, we analyze how to minimize energy consumption per information bit in single link. Further, Target bit error probability, Packet length, Retransmission and Transmission distance for both coded and un coded system are considered as the performance metrics for the optimization of energy. The simulation results illustrate the effect of energy efficient modulation schemes for variable distances and fixed bit error probability.
\end{abstract}

\section{Keywords}

Transceiver, Cooperative Cellular system, Bit error probability, Packet overhead, Adaptive modulation and Energy efficiency.

\section{INTRODUCTION}

The introduction of cooperative networking in Cellular systems has evolved to be the most successful research compared to the implementation of Multiple Input and Multiple Output [MIMO] system [1]. Cooperative wireless networks achieve distributed spatial diversity, wider coverage, low transmit power and reduced interference [2],[3].Selection of subset relays according to performance metric can further enhance the performance of cooperative networking. However, when the energy expenditure of the entire network is considered, lower transmission ranges result in increased number of packets to be forwarded in the network, which in turn increases energy consumption in transceiver circuitries. Although higher transmission power results in higher amplifier energy consumptions, number of forwarded packets is reduced, which in turn results in lower transceiver circuit energy consumption. Therefore, it is essential to consider both the number of packets forwarded in the network and the transceiver energy consumption while designing the strategies.

Majority of the energy saving techniques in the literature focused on the energy minimization for signal transmission [4],[6], and energy consumed by transceiver circuitry are usually neglected because it cannot be comparable with the transmission energy. Energy efficiency of cooperative communication in clustered networks has been investigated in [9]. In [8], it is shown that if the long-haul transmission distance between clusters is large enough, cooperative transmissions can dramatically reduce the total energy consumption even when all the collaboration overhead is considered. Because of the cooperation overhead, the energy 
efficiency of the cooperative communication may degrade with the increase of the number for the cooperators, i,e. more cooperators may not be more energy-efficient[9]. Based on a simple relay selection strategy, the energy efficiency of selective relay cooperation schemes is investigated in [10].

Due to the dynamic nature of the cellular system, the conditions governing the link performance are variable [5]. Adaptive modulation techniques can be used to optimize the energy consumption for various channel conditions. In cooperative cellular network, energy saving via cross-layer design is a promising and necessary means towards energy consumption per bit for end-to-end transmission. In [11], the authors provide an energy consumption model for the PHY layer and minimize the energy consumption per information bit under AWGN channels. In this paper we extend the work to minimize the energy consumption per information bit including the effects of retransmission and packet overhead. We minimize the energy consumption over both bit error probability and packet length.

The rest this paper is organized as follows. The system model including cooperative cellular networking, Conventional transceiver and packet format are discussed in Section II. In Section III, we discuss various parameters to minimize the energy consumption per information bit over target bit error probability and packet length. Numerical results and parameter assumptions with respect to Release 6 are presented in Section IV. We conclude, in Section V.

\section{SYSTEM MODEL}

Figure 1, represents a cooperative cellular system. The system model considered is the Uplink Packet Access [HSUPA]. The end-to-end cooperation is considered from the source mobile equipment [ME] to Base station [BS]. In Phase 1, the source [ME1] broadcasts its information to both the destination [BS] and the relays. In Phase 2, each relay [ME2 \&ME3] helps others by forwarding the information that it receives in Phase 1. If each relay user may decode the received information and forward it (corresponding to the DAF protocol), or simply amplify and forward it (corresponding to the AAF protocol).

Compared with multi- node cooperative schemes, single relay cooperation requires neither cooperative beamforming nor distributed space-time-coding. Selective single relay cooperative schemes are easy to implement and incur less cooperation overhead, and can potentially achieve the same diversity-multiplexing tradeoff as that of multinode cooperative schemes [12]. Hence single-relay-selection cooperative strategies are practically appealing and have also been discussed in [6],[13]. However, most existing work on selective cooperation schemes focus on the multiplexing diversity tradeoff analysis, where a fixed power level is assumed at the source and relays [12]. Power control issues are investigated in [6], [13] from an information theoretic point of view based on the outage probability analysis. Our focus here is on minimization of energy consumption over both bit error probability as well as packet length in single cooperative scheme.

We consider a packet structure, in which we assume $\mathrm{L}_{\mathrm{I}}$ information bits in payload of each packet. The upper layer header contains the control information and packet ID. From the view of the PHY and MAC layers, the payload and the upper layer header are indistinguishable. Therefore, the payload and upper layer header are modulated and coded similarly. The preamble is a certain predefined sequence that serves the purpose of synchronization, Automatic gain controller, etc. The length, modulation type and duration notations are listed in Table I. 


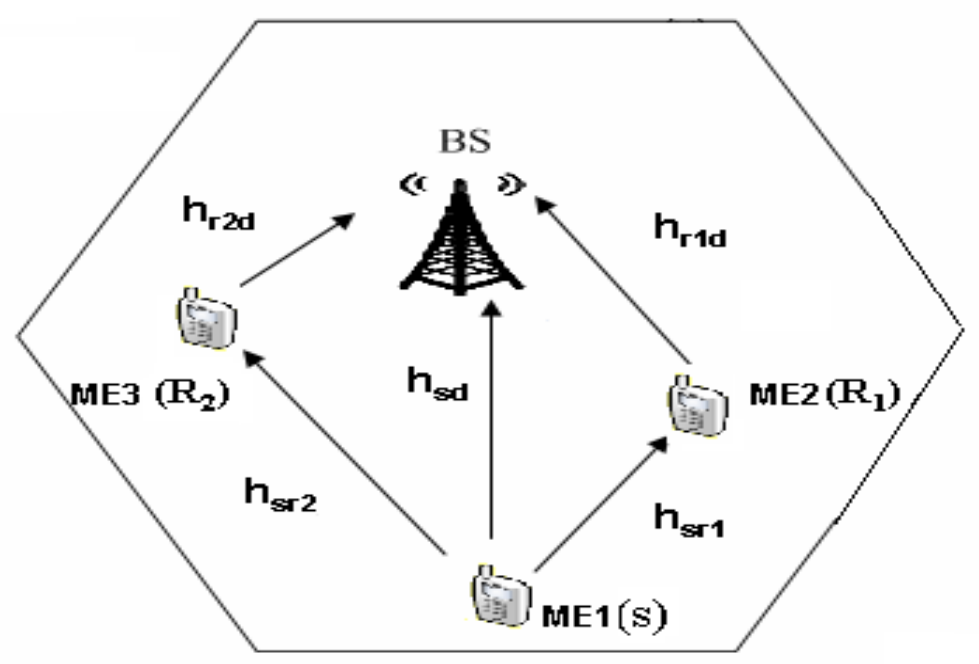

Figure 1. A cooperative cellular system model with High speed uplink packet access.

Table I. Packet Structure Parameters.

\begin{tabular}{|l|l|l|l|}
\hline Component & Length (bits) & Duration (s) & Modulation \\
\hline Payload & $\mathrm{L}_{\mathrm{I}}$ & $\mathrm{T}_{\mathrm{I}}$ & Adaptive \\
\hline Upper layer Header & $\mathrm{L}_{\mathrm{UH}}$ & $\mathrm{T}_{\mathrm{UH}}$ & Adaptive \\
\hline PHY/MAC Header & $\mathrm{L}_{\mathrm{H}}$ & $\mathrm{T}_{\mathrm{H}}$ & $\begin{array}{l}\text { BPSK/Coded } \\
\text { BPSK }\end{array}$ \\
\hline Preamble & - & $\mathrm{T}_{\mathrm{P}}$ & \\
\hline
\end{tabular}

Fig. 2 and 3 represents the transmitter and receiver block independently. At the transmitter end, energy comes from the transmitted energy and the energy consumed in the circuit. At receiver end, energy consumption is considered only for the circuitry. The power consumption by the Digital to Analog converter (DAC), Low Pass Filter (LPF), Band Pass Filter (BPF), Mixer and frequency synthesizer are assumed to be constants. The power consumption of the power amplifier can be expressed as

$$
\mathrm{P}_{\mathrm{amp}}=\beta \mathrm{P}_{\mathrm{t}} \text {. }
$$

Where $\mathrm{P}_{\mathrm{t}}$ is the transmission power and $\beta=\frac{\varepsilon}{\rho}-1, \varepsilon$ is the peak to average ratio and $\rho$ is draining efficiency. Both $\varepsilon$ and $\rho$ are determined by modulation scheme. 
International Journal of Distributed and Parallel Systems (IJDPS) Vol.3, No.5, September 2012

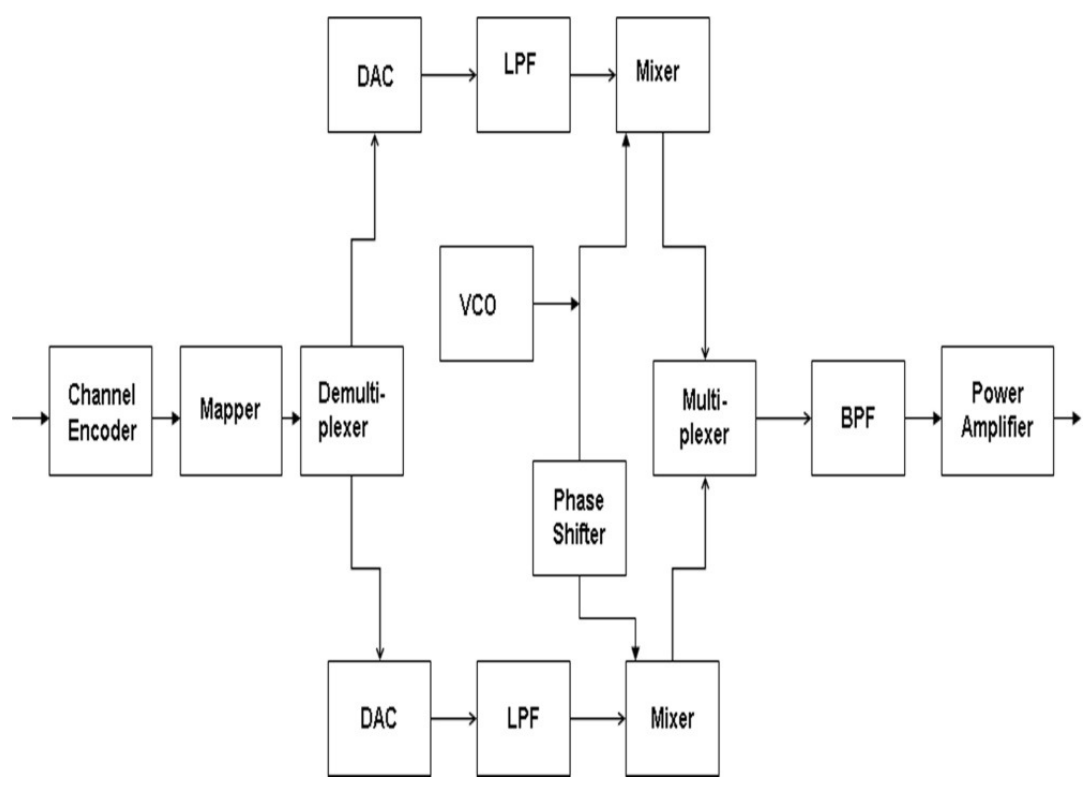

Figure 2. A Conventional transmitter Block diagram.

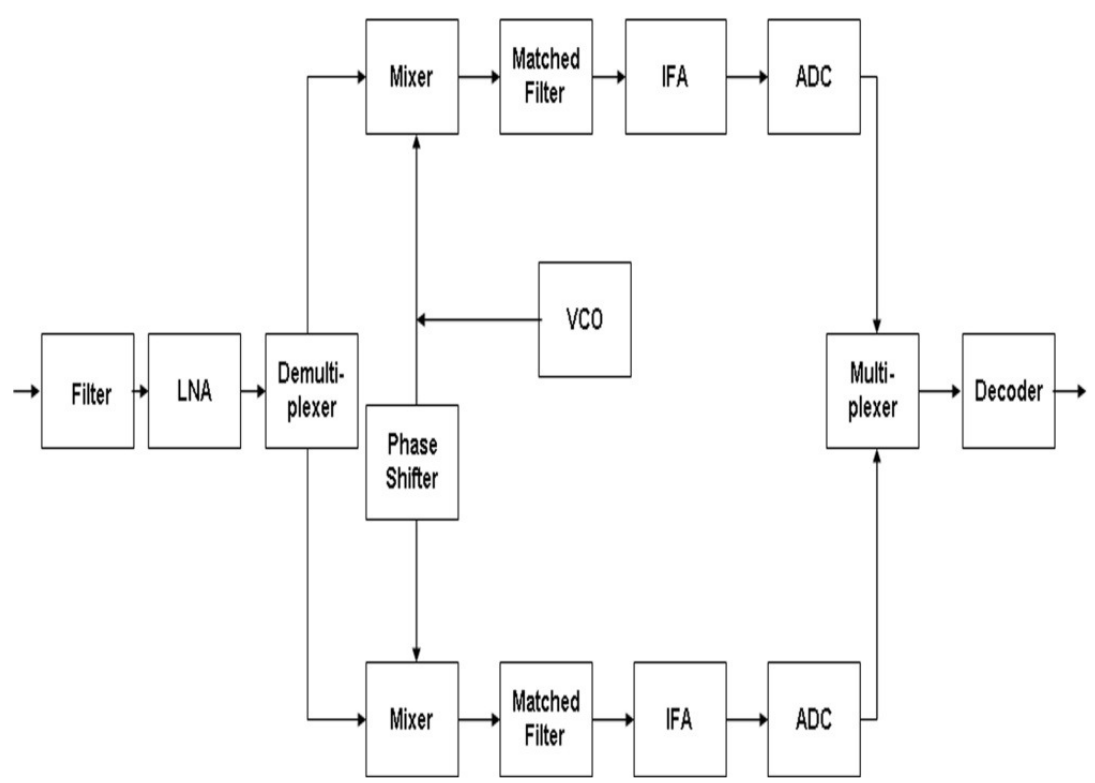

Figure 3. A conventional receiver block diagram.

\section{MINIMIZATION OF ENERGY CONSUMPTION}

\subsection{Energy Consumption per Packet}

We assume the transmitter and receiver will remain in ON state for $\mathrm{T}_{\text {on }}$ seconds,

$$
\text { where } \mathrm{T}_{\mathrm{On}}=\left(\mathrm{T}_{\mathrm{I}}+\mathrm{T}_{\mathrm{UH}}+\mathrm{T}_{\mathrm{H}}\right) / \mathrm{R}_{\mathrm{C}}+\mathrm{T}_{\mathrm{P}}
$$

$R_{c}$ is the channel code rate and is set to 1 for uncoded system. The total energy consumption required to transmit or receive $\mathrm{L}_{\mathrm{L}}$ information bits is 
International Journal of Distributed and Parallel Systems (IJDPS) Vol.3, No.5, September 2012

$$
\mathrm{E}=\left(\mathrm{P}_{\mathrm{t}} / \mathrm{G}_{\mathrm{c}}+\mathrm{P}_{\mathrm{amp}}+\mathrm{P}_{\mathrm{c}}\right) \mathrm{T}_{\mathrm{on}} \text {. }
$$

Where $P_{t}$ is the transmitted power used in an uncoded system, $G_{c}$ is the coding gain, $P_{a m p}=\beta P_{t}$ is the power consumption of the power amplifier, and $P_{c}$ power consumption of circuit components of receiver and transmitter. The Trasnsmit power, $\mathrm{P}_{\mathrm{t}}$ can be determined from the SNR $(\gamma)$ at the receiver and bit error probability $P_{b}$. The SNR per symbol is defined as $\gamma=P_{r} /\left(2 \mathrm{BN}_{0}\right)$, where $\mathrm{P}_{\mathrm{r}}$ is the received power, $\mathrm{B}$ is signal bandwidth and Noise power spectrum density. It is easy to find $\gamma=\mathrm{f}\left(\mathrm{P}_{\mathrm{b}}\right)$ by suing exponential approximation to the Qfunction (Table II).

Table II. Bit error probability and Bandwidth Efficiency for Coherent Modulations/ Demodulations.

\begin{tabular}{|l|c|l|}
\hline Modulation & $\mathrm{P}_{\mathrm{b}}(\gamma)$ & $\eta($ bits/Hertz) \\
\hline BPSK & $\mathrm{P}_{\mathrm{b}} \approx \frac{1}{2} \mathrm{e}^{-\gamma}$ & $\eta=1$ \\
\hline MPSK & $\mathrm{P}_{\mathrm{b}} \approx \frac{1}{\log _{2}^{\mathrm{M}}} \mathrm{e}^{-\gamma \sin ^{2}\left(\frac{\pi}{\mathrm{M}}\right)}$ & $\eta=\log _{2}^{\mathrm{M}}$ \\
\hline MQAM & $\mathrm{P}_{\mathrm{b}} \approx \frac{2}{\log _{2}^{\mathrm{M}}} \mathrm{e}^{-\frac{3}{2(\mathrm{M}-1)} \gamma}$ & $\eta=\log _{2}^{\mathrm{M}}$ \\
\hline MFSK & $\mathrm{P}_{\mathrm{b}} \approx \frac{\mathrm{M}-1}{2 \log _{2}^{\mathrm{M}}} \mathrm{e}^{-\frac{1}{2}}$ & $\eta=\frac{2 \log _{2}^{\mathrm{M}}}{\mathrm{M}}$ \\
\hline
\end{tabular}

Based on the signal propagation model, $\mathrm{P}_{\mathrm{t}}=\mathrm{GP}_{\mathrm{r}}$. Where $\mathrm{G} \sim \mathrm{d}^{3.5}$ represents the path loss. Therefore the transmitted power can be eventually denoted as:

$$
\mathrm{P}_{\mathrm{t}}=2 \mathrm{BN}_{0} \mathrm{G} \gamma
$$

The power consumption of the circuit components of the transmitted and the receiver is defines as

$$
\mathrm{P}_{\mathrm{c}}=2 \mathrm{p}_{\text {mixer }}+2 \mathrm{P}_{\text {syn }}+\mathrm{P}_{\text {filter }}+\mathrm{P}_{\mathrm{DAC}}+\mathrm{P}_{\mathrm{ADC}}+\mathrm{P}_{\mathrm{v}}
$$

The parameters of the above equation are chosen based on the typical implementation of High speed Uplink Packet Access, Release 6 (category-6), specified under Table III.

Energy consumption for transmit packet containing $L_{I}$ information bits is given as:

$$
\mathrm{E}=(1+\beta) 2 \mathrm{BN}_{0} \mathrm{G} \gamma T_{\text {on }} / \mathrm{G}_{\mathrm{c}}+\mathrm{P}_{\mathrm{c}} \mathrm{T}_{\text {on }}
$$


International Journal of Distributed and Parallel Systems (IJDPS) Vol.3, No.5, September 2012

Table III. Power Consumption Details

\begin{tabular}{|c|c|c|c|c|c|}
\hline & $\mathbf{P}_{\text {filter }}$ & $\mathbf{P}_{\text {mixer }}$ & $\mathbf{P}_{\text {amp }}$ & $\mathbf{P}_{\text {LNA }}$ & $\mathbf{P}_{\text {syn }}$ \\
\hline $\begin{array}{c}\text { Transmitter, } \\
\mathbf{P}_{\text {ct }}\end{array}$ & $2.5 \mathrm{~mW}$ & $30.3 \mathrm{~mW}$ & $\beta \mathrm{P}_{\mathrm{t}}$ & - & $50 \mathrm{~mW}$ \\
\hline Receiver, $\mathbf{P}_{\text {cr }}$ & $2.5 \mathrm{~mW}$ & $30.3 \mathrm{~mW}$ & - & $20 \mathrm{~mW}$ & $50 \mathrm{~mW}$ \\
\hline
\end{tabular}

\subsection{Optimization of Energy Consumption per Packet:}

Whenever there is a bit error in the received packet, a retransmission is required. For a packet containing $\mathrm{L}_{\mathrm{I}}$ information bits, the probability of packet error is

$$
\mathrm{P}_{\mathrm{pc}}=1-\left(1-\mathrm{P}_{\mathrm{b}}\right)^{\mathrm{L}_{\mathrm{L}}}{ }^{+\mathrm{L}} \mathrm{UH}
$$

Because of the robust modulation scheme use for PHY/MAC header, we assume the probability of error occurrence is low. Considering retransmission scheme for the packet errors, before the transmitter and receiver will spent $T_{\text {tr }}$ seconds to switch between states(OFF $\left.\rightarrow O N\right)$, the time taken to start up the frequency synthesizer $T_{t r}=5 \mu$ s and the power consumption of $P_{t r}$ if equal to $\mathrm{P}_{\mathrm{syn}}$, as per Table III. $\mathrm{T}_{\mathrm{ACK}}$ is time period of transmitter to listen for an acknowledgement, $\mathrm{T}_{\mathrm{ACK}} \approx \frac{\mathrm{L}_{\mathrm{H}}}{\mathrm{BR}_{\mathrm{C}}}+\mathrm{T}_{\mathrm{P}}$.

Energy consumption during each time period can be calculated as:

$$
\begin{aligned}
& \mathrm{E}_{\text {tr }}=\mathrm{P}_{\text {syn }} \mathrm{T}_{\text {tr }} \\
& \mathrm{E}_{\text {IPS }}=\mathrm{P}_{\text {Syn }} \mathrm{T}_{\text {IPS }} \text {, IPS is Inter packet space } \\
& \mathrm{E}_{\mathrm{LN}}=\left(\mathrm{P}_{\mathrm{Cr}}-\mathrm{P}_{\mathrm{V}}\right) \mathrm{T}_{\mathrm{ACK}} \\
& \mathrm{E}_{\mathrm{ACK}}=\mathrm{P}_{\mathrm{Cr}} \mathrm{T}_{\mathrm{ACK}} \\
& \mathrm{E}_{\mathrm{tx}}=\left\lfloor(1+\beta) 2 \mathrm{BN}_{0} \mathrm{G} \gamma / \mathrm{G}_{\mathrm{c}}+\mathrm{P}_{\mathrm{ct}}\right\rfloor \mathrm{T}_{\text {on }}
\end{aligned}
$$

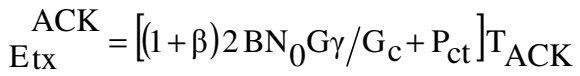

$$
\begin{aligned}
& \mathrm{E}_{\mathrm{rx}}=\mathrm{P}_{\text {cr }} \mathrm{T}_{\mathrm{on}}
\end{aligned}
$$

In the first $(\mathrm{m}-1)$ transmissions, the energy consumption during the $\mathrm{T}_{\mathrm{ACK}}$ period at the transmitter is $\mathrm{E}_{\mathrm{LN}}$, since the transmitter does not receive an acknowledgment from the receiver and decoding is not needed. ACK is the energy consumption of transmitting the acknowledgment after receiving the $\mathrm{m}^{\text {th }}$ packet. We assume that in the first $T_{A C K}$ periods, the energy consumption at the receiver is zero. Therefore, the total transmitter and receiver energy consumption of the $m$ deliveries are

$$
\begin{aligned}
& \mathrm{E}_{\mathrm{t}}(\mathrm{m})=\left(2 \mathrm{E}_{\mathrm{IPS}}+\mathrm{E}_{\mathrm{tx}}+\mathrm{E}_{\mathrm{LN}}\right)(\mathrm{m}-1)+2 \mathrm{E}_{\mathrm{tr}} \\
& +2 \mathrm{E}_{\mathrm{tr}}+2 \mathrm{E}_{\mathrm{IPS}}+\mathrm{E}_{\mathrm{tx}}+\mathrm{E}_{\mathrm{ACK}} \\
& \mathrm{E}_{\mathrm{r}}(\mathrm{m})=\left(2 \mathrm{E}_{\mathrm{IPS}}+\mathrm{E}_{\mathrm{tx}}\right) \mathrm{m}+2 \mathrm{E}_{\mathrm{tr}}+\mathrm{E}_{\mathrm{tx}}^{\mathrm{ACK}}
\end{aligned}
$$

Consequently, to successfully deliver a packet, the average energy consumption is 
International Journal of Distributed and Parallel Systems (IJDPS) Vol.3, No.5, September 2012

$$
\overline{\mathrm{E}}=\sum_{\mathrm{i}=1}^{\infty}\left[\mathrm{E}_{\mathrm{t}}(\mathrm{i})+\mathrm{E}_{\mathrm{r}}(\mathrm{i})\right] \mathrm{P}_{\mathrm{r}}\{\mathrm{m}=\mathrm{i}\}
$$

Where $m$ is the number of transmissions and $\mathrm{P}_{\mathrm{r}}\{\mathrm{m}=\mathrm{i}\}$ denotes the probability that the number of transmissions equals $i$, which is given as $\mathrm{P}_{\mathrm{r}}\{\mathrm{m}=\mathrm{i}\}=\mathrm{P}_{\mathrm{Pe}}{ }^{i-1}\left(1-\mathrm{P}_{\mathrm{Pe}}\right)$. After simplification, we have

$$
\begin{aligned}
\overline{\mathrm{E}} \approx & \frac{\left(2 \mathrm{E}_{\mathrm{IPS}}+\mathrm{E}_{\mathrm{tx}}+\mathrm{E}_{\mathrm{LN}}\right)}{1-\mathrm{P}_{\mathrm{pe}}}+2 \mathrm{E}_{\mathrm{tr}}+\mathrm{P}_{\mathrm{v}} \mathrm{T}_{\mathrm{ACK}} \\
& +\frac{\left(2 \mathrm{E}_{\mathrm{IPS}}+\mathrm{E}_{\mathrm{rX}}\right)}{1-\mathrm{P}_{\mathrm{pe}}}+2 \mathrm{E}_{\mathrm{tr}}+\mathrm{E}_{\mathrm{tx}} \mathrm{ACK}
\end{aligned}
$$

Each packet contains $\mathrm{L}_{\mathrm{I}}$ information bits. Therefore, the average energy consumption per information bit is

$$
\overline{\mathrm{E}}_{\mathrm{bit}}=\frac{\overline{\mathrm{E}}}{\mathrm{L}_{\mathrm{I}}}
$$

To minimize $\overline{\mathrm{E}}_{\text {bit }}$ with respect $\mathrm{L}_{\mathrm{I}}$, we set $\frac{\partial \overline{\mathrm{E}}_{\text {bit }}}{\partial \mathrm{L}_{\mathrm{I}}}=0$ which is simplified as

$$
\mathrm{A}_{1} \mathrm{~L}^{2} \mathrm{I}+\mathrm{B}_{1} \mathrm{~L}_{\mathrm{I}}+\mathrm{C}_{1}=0
$$

Where

$$
\begin{aligned}
& \mathrm{A}_{1}=\frac{\mathrm{P}_{\mathrm{on}} \mathrm{P}_{\mathrm{b}}}{\mathrm{B}_{\eta}} \\
& \mathrm{B}_{1}=\mathrm{P}_{\mathrm{b}}\left(4 \mathrm{E}_{\mathrm{IPS}}+\mathrm{E}_{\mathrm{LN}}+\mathrm{P}_{\mathrm{on}} \mathrm{T}_{\mathrm{p}}+\frac{\mathrm{P}_{\mathrm{On}} \mathrm{L}_{\mathrm{H}}}{\mathrm{BR}_{\mathrm{c}}}+\frac{\mathrm{P}_{\mathrm{On}} \mathrm{L}_{\mathrm{UH}}}{\mathrm{B \eta}_{\mathrm{c}}}\right) \\
& \mathrm{C}_{1}=-\left(4 \mathrm{E}_{\mathrm{IPS}}+\mathrm{E}_{\mathrm{LN}}+4 \mathrm{E}_{\mathrm{tr}}+\mathrm{E}_{\mathrm{tx}}^{\mathrm{ACK}}\right. \\
& \left.+\mathrm{P}_{\mathrm{V}} \mathrm{T}_{\mathrm{ACK}}+\mathrm{P}_{\mathrm{On}} \mathrm{T}_{\mathrm{p}}+\frac{\mathrm{P}_{\mathrm{on}} \mathrm{L}_{\mathrm{H}}}{\mathrm{BR}_{\mathrm{c}}}+\frac{\mathrm{P}_{\mathrm{On}} \mathrm{L}_{\mathrm{UH}}}{\mathrm{B \eta} \mathrm{R}_{\mathrm{c}}}\right)
\end{aligned}
$$

With $P_{\text {on }}=2(1+\beta) B N_{0} G \gamma / G_{c}+P_{c}$.Solving (9) yields the optimum number of information bits per packet, $\mathrm{L}_{\mathrm{I}}$

$$
\mathrm{L}_{\mathrm{I}}^{*}=\frac{-\mathrm{B}_{1}+\sqrt{\mathrm{B}_{1}^{2}-4 \mathrm{~A}_{1} \mathrm{CL}_{1}}}{2 \mathrm{~A}_{1}}
$$

Correspondingly, the optimum target $\mathrm{P}_{\mathrm{b}}$ can be found by solving. The closed form solution of the optimum target $\mathrm{P}_{\mathrm{b}}$ can be found through the following approximation (for small $\mathrm{P}_{\mathrm{b}}$ )

When using M-QAM,

$$
\begin{aligned}
& \mathrm{P}_{\mathrm{b}} \ln \mathrm{P}_{\mathrm{b}} \approx \mathrm{P}_{\mathrm{b}} \mathrm{P}_{-1} \\
& \mathrm{P}_{\mathrm{b}} \mathrm{P}_{-10 \mathrm{P}_{\mathrm{b}}+1}
\end{aligned}
$$


International Journal of Distributed and Parallel Systems (IJDPS) Vol.3, No.5, September 2012

$$
\mathrm{P}_{\mathrm{b}} \approx \frac{1}{1+\left(\mathrm{L}_{\mathrm{I}}+\mathrm{L}_{\mathrm{UH}}\right)\left[\ln \left(\frac{2}{\mathrm{~b}}\right)+10+\frac{\mathrm{P}_{\mathrm{c}} \mathrm{T}_{\mathrm{On}}+4 \mathrm{E}_{\mathrm{IPS}}+\mathrm{E}_{\mathrm{LN}}}{\frac{2}{3}\left(2^{\mathrm{b}}-1\right) \mathrm{A}_{2}}\right]}
$$

Where $\mathrm{A}_{2}=\frac{(1+\beta) 2 \mathrm{BN}_{0} \mathrm{G}(\mathrm{d}) \mathrm{T}_{\mathrm{on}}}{\mathrm{G}_{\mathrm{c}}}$

When the transmission distance $\mathrm{d}$ is large, $\mathrm{A}_{2} \approx \infty$.

$$
\mathrm{P}_{\mathrm{b}}^{*} \approx \frac{1}{1+\left(\mathrm{L}_{\mathrm{I}}+\mathrm{L}_{\mathrm{UH}}\right)\left[\ln \left(\frac{2}{\mathrm{~b}}\right)+10\right]}
$$

Therefore, the target bit error probability will eventually converge to a value solely determinate by the packet length and the modulation scheme. The optimum target bit error probabilities of other modulation schemes and their corresponding convergence values can be obtained similarly. Furthermore, equation (11) reveals a one-to-one relation between $\mathrm{P}_{\mathrm{b}}^{*}$ and $\mathrm{L}_{\mathrm{I}}^{*}$ at any given distance.

\section{NUMERICAL RESULTS}

For our simulation results and theoretical calculations we assume High Speed Uplink packet Access (HSUPA) under Release 6 (Category-6). Bandwidth of $2000 \mathrm{MHz}, \mathrm{L}_{\mathrm{UH}}=160$ bits, $\mathrm{L}_{\mathrm{H}}=$ 32 bits, $T_{P}=2 \mathrm{~ms}, R_{c}=1 / 3, G_{c}=4.2$, transmitter power of UE $=21 \mathrm{dBm}$ (Class $4 \mathrm{UE}$ ) and the packet length, $\mathrm{L}_{\mathrm{I}}$ is limited to 11520 bits.

Figure 4and 5 represents the increase in the Optimum target bit error probability at different transmission distances. This is because, as the transmission distance increases, a higher target $P_{b}$ is preferred lest as the transmission energy increases to mitigate the path loss.

The optimum total energy consumption per information bit at different transmission distances is shown in Figure 6. As the transmission distance increases the energy per bit increases. As shown in Figure6, uncoded 4-QAM, uncoded 8PSK, uncoded QPSK and Coded QPSK are preferred for short, medium and long distance respectively. At shorter transmission distances, the energy consumption is effected by the energy consumption of the circuitry. On other hand, at longer distances, the energy consumption is dominated by the transmission energy. Hence, modulation and coding schemes that require lower SNR will have an advantage. Figure 7, depicts the energy consumption for information bit for various packet sizes under different header sizes. To reduce the retransmission cost for shorter distances transmission, a shorter packet length is preferred. 
International Journal of Distributed and Parallel Systems (IJDPS) Vol.3, No.5, September 2012

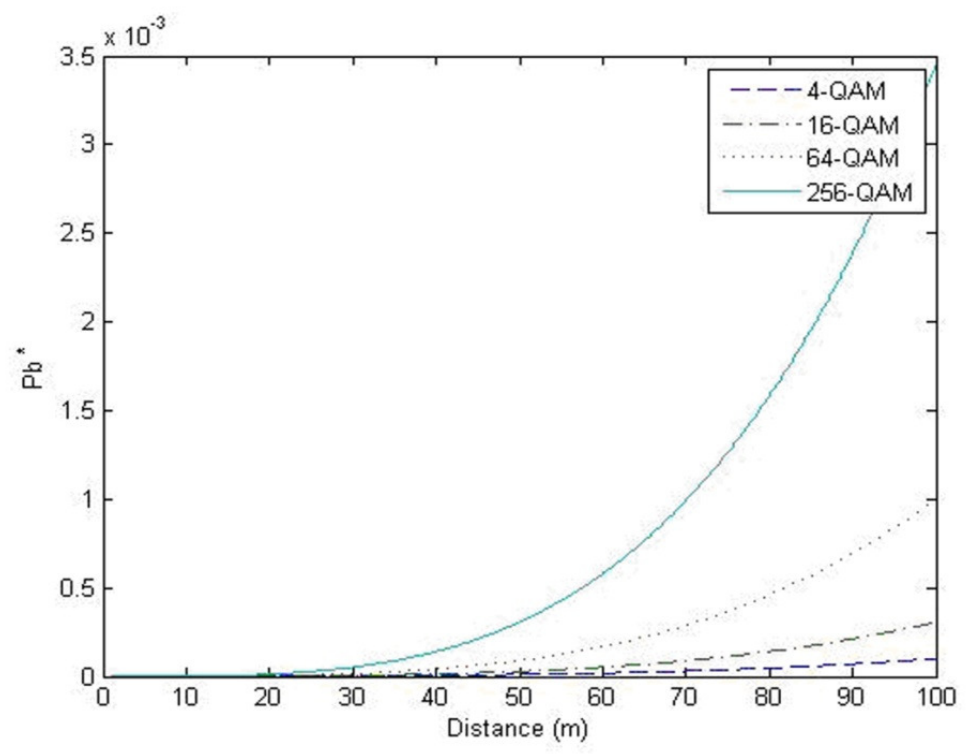

Figure 4. Optimum target bit error Probability Vs Distance, for higher order modulation schemes.

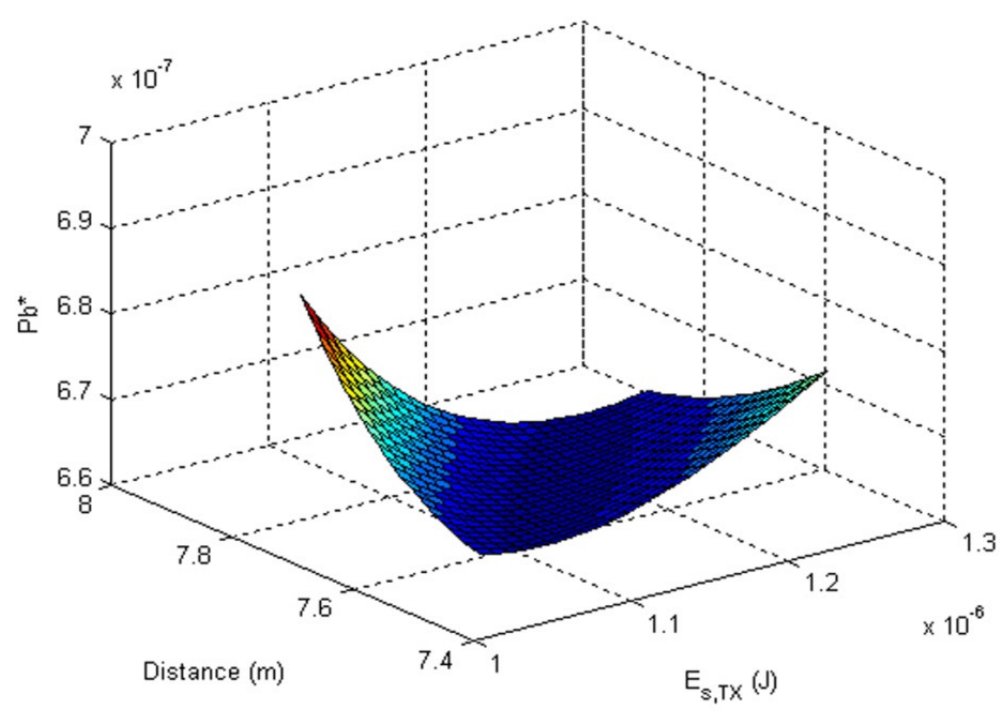

Figure 5. Depicting the Optimum energy consumption per bit, Distance and Optimum target error probability for 4-QAM. 
International Journal of Distributed and Parallel Systems (IJDPS) Vol.3, No.5, September 2012

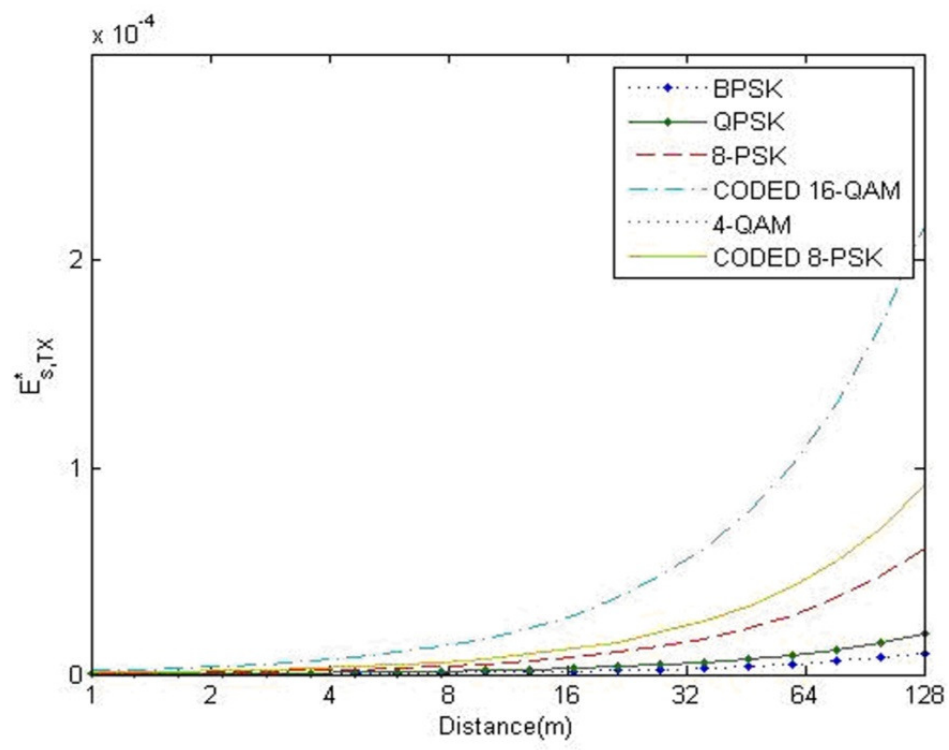

Figure 6. Optimum energy consumption per information bit for various distances.

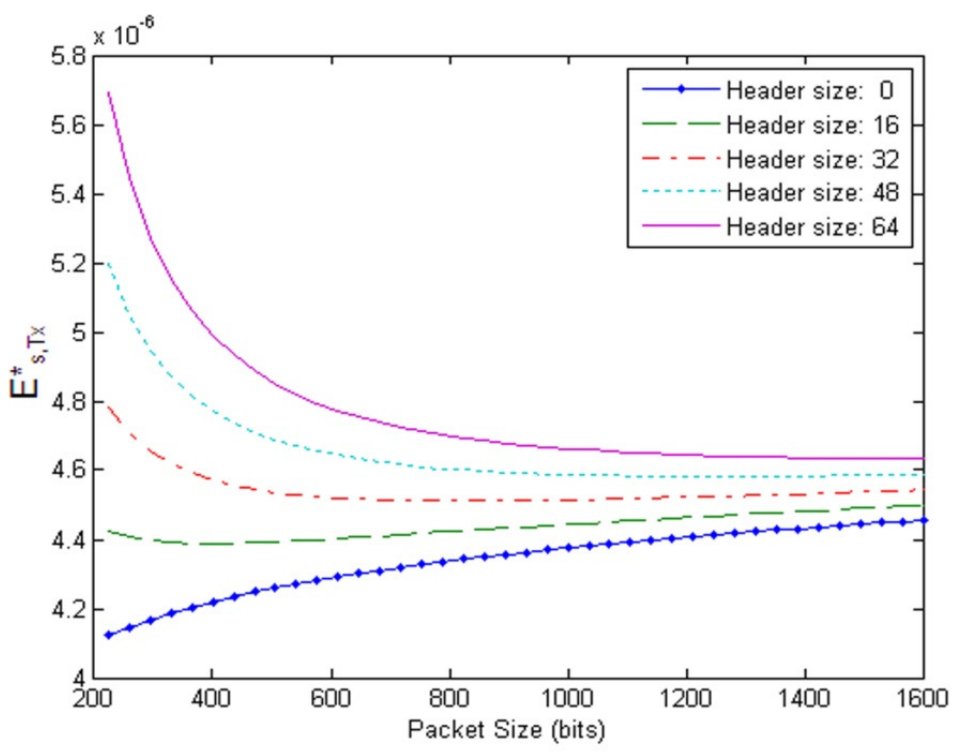

Figure 7. The energy consumption per information bit for various packet sizes.

\section{CONCLUSION}

In this paper, we emphasize the energy consumption per information bit under cooperative uplink cellular systems. An optimization over both target bit error probability and packet length is preferred with consideration of retransmission. We studied the effect of circuitry for shorter distance transmissions. The analysis is performed over both coded and un coded modulation schemes. For more energy efficient transmission for short distances, the system should adopt large packet length, small target bit error probability and high bandwidth efficient modulation 
International Journal of Distributed and Parallel Systems (IJDPS) Vol.3, No.5, September 2012

schemes. Further, as the transmission distance increases, a flattening of the optimum values of packet length and target bit error probability is observed.

\section{REFERENCES}

[1] M. Nokleby, and B. Aazhang, (2010) "User Cooperation for Energy-Efficient Cellular Communications," IEEE International Conference on Communications (ICC) 2010, pp.1-5, 2327.

[2] B. Kan, L. Cai, L. Zhao, Y. Xu, (2007) "Energy efficient design of WSN based on an accurate power consumption model,” IEEE WiCom'07, Shanghai, China, pp. 2751-2754.

[3] J.N.Laneman, D.N.C.Tse, and G.W.Wornell, (2004) "Cooperative diversity in wireless networks: Efficient protocols and outage behavior," IEEE trans. Inf. Theory, vol. 50, no. 12, pp. 30623080.

[4] Schaar M, Turaga D.S., (2007) "Cross-layer packetization and retransmission strategies for delaysensitive wireless multimedia transmission,” IEEE Trans. Multimedia, pp. 185-197.

[5] Madan R, Mehta N B, Molisch A F, et al. (2008) "Energy-efficient cooperative relaying over fading channels with simple relay selection," IEEE Transaction on Wireless Communications, pp.3013-3025

[6] N.Ahmed, M.A. Khojastepour, A. Sabharwal and B. Aazhang, (2006) "Outage minimization with limited feedback for the fading relay channels," IEEE Trans. Commun. Vol. 54, no. 4, pp. 659669.

[7] R. Rost, and G. Fettweis, (2010) "Green communication in cellular network with fixed relay nodes," Cooperative Cellular Wireless Communications," published by Cambridge university press.

[8] Aghdasi H.S., Abbaspour M. (2008) "ET-MAC: An Energy-efficient and High Throughput MAC Protocol for Wireless Sensor Networks," In Proceedings of the Communication Networks and Services Research Conference, Halifax, Canada, pp. 526-532.

[9] A. Vosoughi, and Y. Jia, (2010) "Maximizing Throughput in Cooperative Networks via Crosslayer Adaptive Designs,” Proc. of IEEE Sarn off Symposium'10, pp. 1-6.

[10] S.Cui, A. Z.Goldsmith, and A.Bahai, (2005) "Energy Constrained Modulation Optimization", IEEE Transactions on wireless Communications, vol.4, no. 8, pp. 2349-2360.

[11] A.Bletsas, A.Khitsi, D.P.Reed, and A.Lippman, (2006) "A Simple cooperative diversity method based on Network path selection,” IEEE J. Select. Areas Commun., vol. 24, no. 3, pp. 659-672.

[12] Asif D. Gandhi, and Mark E. Newbury, (2011) "Evaluation of the energy efficiency metrics for wireless networks", Bell Labs Technical Journal, vol. 16, issue 1, pp. 207-215.

\section{Authors}

M.SUSHANTH BABU received his B.E. in Electronics and Communication Engineering in 2002 from North Maharastra University and his M.Tech. degree from Jawaharlal Nehru Technological University, Hyderabad in 2008. He has been working towards his Ph.D. degree in Wireless Communications at Jawaharlal Nehru Technological University, Hyderabad since 2009. He is presently working as Associate Professor in Department of Electronics and Communication Engineering. He guided 18 Masters and $30 \mathrm{UG}$ projects. He

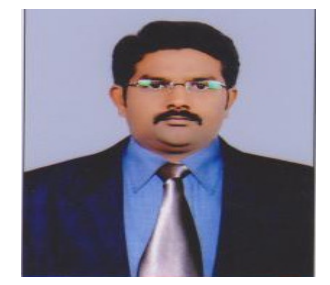
is a member of professional bodies like, IEEE, ISTE and IETE. He is technical program committee member of 12 IEEE International Conferences. His research interests are in the areas of Wireless Mobile Communication, Cellular Networking, Distributed Cooperative Communication, MIMO and Signal Processing Applications. 
International Journal of Distributed and Parallel Systems (IJDPS) Vol.3, No.5, September 2012

Prof.K.KISHAN RAO is currently a Professor in Electronics and Communication Engineering and working as Director in Viswambhara Educational Society. He received his B.E. and M.E. degrees from Osmania University in 1965 and 1967.He is awarded with Ph.D. degree from Indian Institute of Technology, Kanpur [IIT] in 1973.He worked as Principal for National Institute of Technology and Kakathiya Institute of Technology and Science, Warangal. He a senior member of professional bodies like IEEE

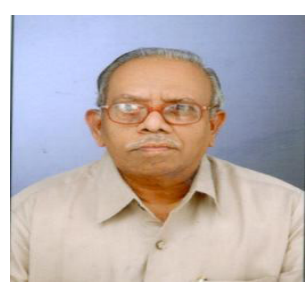
[comsoc], ISTE, and IETE. He has published over 78 International articles. He currently serves as Editor for International Journal of Wireless Personal communication, Springer and International Journal of Wireless Networks, Springer. He guided 03 Ph.D scholars and guided 62 Master Projects. His research interests are in the areas of Wireless Communications, Signal Processing Applications and Cooperative Mobile Communications. 